\title{
Upstream magnetospheric ion flux tube within a magnetic cloud: Wind/STICS
}

\author{
Arik Posner \\ Institut für Experimentelle und Angewandte Physik, Universität Kiel, Germany \\ Michael W. Liemohn and Thomas H. Zurbuchen \\ Department of Atmospheric, Oceanic and Space Sciences, University of Michigan, USA \\ Received 16 August 2002; revised 6 January 2003; accepted 10 February 2003; published 29 March 2003.
}

[1] We present a case study of a remarkable upstream $\mathrm{O}^{+}$ and $\mathrm{N}^{+}$ion outflow event. We present observational evidence for spatially structured outflow of these Low Charge State Heavy Ions (LCSHIs) of magnetospheric origin along a small reconnected field line region within the framework of a magnetic cloud of an ICME. From the particles' in situ 3D distribution function we conclude that in this case the interaction of the outflow with the bow shock is small. We conclude further that the gyrophases of outflowing ions at the reconnection point are randomly distributed. This leads to the formation of a flux tube with a specific geometry. In particular, the outflow reveals spatial dispersion and non-gyrotropy. This result has implications for the size of the dayside reconnection site. INDEX TERMS: 2116 Interplanetary Physics: Energetic particles, planetary; 2111 Interplanetary Physics: Ejecta, driver gases, and magnetic clouds; 2778 Magnetospheric Physics: Ring current; 2784 Magnetospheric Physics: Solar wind/magnetosphere interactions. Citation: Posner, A., M. W. Liemohn, and T. H. Zurbuchen, Upstream magnetospheric ion flux tube within a magnetic cloud: Wind/STICS, Geophys. Res. Lett., 30(6), 1346, doi:10.1029/2002GL016116, 2003.

\section{Introduction}

[2] The discovery of suprathermal $\mathrm{O}^{+}$ions in the solar wind upstream of the Earth's bow shock by Möbius et al. [1986] dates back to observations made in 1980 with composition instrumentation on AMPTE. For a long time it was debated to what extent magnetospheric particles, easily identified by their low charge state compared to solar wind ions, contribute to the upstream particle populations. It is well known that particles in the magnetosphere are accelerated to energies high enough to outrun the incoming solar wind flow. However, bow shock acceleration and reflection is another source of suprathermal particles with that capability.

[3] The charge state and elemental composition are key observations to resolve the dispute. Recently, Christon et al. [2000] showed that in Geotail/EPIC/STICS [Williams et al., 1994] observations, periods of upstream $\mathrm{O}^{+}$and $\mathrm{N}^{+}$below $20 \mathrm{keV} / \mathrm{n}$ are more common than previously thought. However, according to observations with Wind/STEP [Desai et al., 2000] the average elemental composition of upstream $\mathrm{C}, \mathrm{N}$, and $\mathrm{O}$ above $30 \mathrm{keV} / \mathrm{n}$ resembles that of the solar wind.

Copyright 2003 by the American Geophysical Union. 0094-8276/03/2002GL016116
[4] Posner et al. [2002] have shown with another STICS instrument that singly charged oxygen and nitrogen ions originating in the Earth's magnetosphere propagate far upstream (at least $150 \mathrm{R}_{\mathrm{e}}$ from the Earth's bow shock). These observations from the orbit of the Wind spacecraft revealed for the first time the boundary conditions for the occurence of upstream magnetospheric ions. Local interplanetary magnetic field (IMF) conditions during such events are a function of the relative positions of Wind and the Earth, revealing that a direct magnetic connection from the spacecraft to the bow shock/magnetosphere has to be established. Most events are observed north of the ecliptic plane with a southward IMF during moderate or strong geomagnetic activity. This finding points towards magnetic reconnection as the most-likely scenario for magnetospheric outflow losses. A strong dependence of the occurence rate of particle events containing magnetospheric origin ions on the solar cycle with an increase of an order of magnitude in frequency from solar minimum to maximum, is also found. This can be attributed to the solar cycle dependence of the magnetospheric $\mathrm{O}^{+}$abundance [Young et al., 1982].

[5] The study focused on observations of series of events. A series is a group of individual bursts of LCSHIs occuring within a few hours of each other. These series of events form a sub-class of magnetospheric outflows upstream of the bow shock that typically occur around the equinoxes and most-likely can be attributed to HILDCAAs (HighIntensity, Long-Duration, Continuous AE Activity) [Tsurutani and Gonzalez, 1987]. In the fast solar wind, where series of events typically occur, Alfvén waves themselves produce short periods of southward IMF at the Earth during which outflow can occur. The variability in the magnetic field associated with these waves makes it very likely that a spacecraft upstream of the bow shock will encounter these shortlived flux tubes of magnetospheric outflow during a single fast solar wind stream.

[6] For this study, we analyze an isolated, high-intensity event that does not fit into the scheme characterized in the above study. We will show that such isolated events form another sub-class of magnetospheric outflow events upstream of the bow shock. These events are more likely to be observed strongly northward of the ecliptic plane upstream of the bow shock.

\section{Instrumentation}

[7] Our study is based on observations from the STICS instrument onboard Wind. Elemental and charge state 
composition is revealed by measuring the combination of energy per charge, time-of-flight, and total energy for each incoming ion. As a part of the SMS package [see Gloeckler et al., 1995, for technical details], STICS covers the full 3D distribution function of ions in the suprathermal (6.2-223.1 $\mathrm{keV} / \mathrm{e})$ range encountered in near-Earth space. The orbit of Wind is constantly modified in order to cover the full range of clock angles about the Earth with distances between roughly 5 and $200 \mathrm{R}_{\mathrm{e}}$. The identification and background reduction procedures for $\mathrm{O}^{+}$and $\mathrm{N}^{+}$magnetospheric ion events are adapted from and fully described in Posner et al. [2002].

\section{Observations}

[8] Figure 1 shows the observations of LCSHIs in the mass-per-charge (m/q) range 10 to $20 \mathrm{amu} / \mathrm{e}$ (first and second panel) during a time period of two days, beginning on Feb. 12, 2000, along with the Dst-index (second panel), in situ magnetic field observations from Wind/MFI [Lepping et al., 1995] (third panel), and the solar wind speed and densities from Wind/SWE [Ogilvie et al., 1995] (bottom panel). On that day at approximately 1654 UT the Wind spacecraft, located almost exactly sunward upstream from the Earth, at a distance of $130 \mathrm{R}_{\mathrm{e}}$, became embedded in the magnetic cloud of an interplanetary coronal mass ejection (ICME). The identification of the ICME and magnetic cloud were made with Wind/MFI (R. Lepping and A. Szabo, GSFC, personal communication, 2002) by fitting a force free flux rope model [Lepping et al., 1990], and ACE/ SWICS data by Lepri et al. [2001], using charge states of Fe ions. A rather smooth rotation in the magnetic field is observed over the time period from 1654 UT on Feb 12 until 0036UT on Feb 13. Low turbulence levels are typical for magnetic clouds, allowing for almost scatter-free transport of energetic particles. On 1900 UT on Feb 12 the interplanetary magnetic field is pointing almost radially inward towards the Sun. Shortly thereafter the number density of LCSHIs reaches the highest value observed outside the magnetosphere/magnetosheath for the whole Wind mission so far. The composition reveals $\mathrm{O}^{+}$and $\mathrm{N}^{+}$ ions with total energies typically above $50 \mathrm{keV}$. Note that a possible contribution of $\mathrm{m} / \mathrm{q}$ above 16 is likely, which would point toward a molecular ion component like $\mathrm{OH}^{+}$ or $\mathrm{H}_{2} \mathrm{O}^{+}$. This observation coincides with the main recovery phase of the Earth's ring current as indicated by the Dst index. The recovery of the ring current happens mainly due to losses of ring current ions on the dayside boundary layer and through charge exchange with the geocorona [Liemohn et al., 1999].

[9] On the left hand side of Figure 2 the period of the Wind/STICS LCSHI event is shown in highest time resolution available ( 3 mins). Individual ions are shown in a scatter plot representation with $\mathrm{m} / \mathrm{q}$ over time. The color coding is chosen according to the E/q scale (presumably $\mathrm{q}=1$ ) to the right. The red and green open histograms indicate the numbers of ions for each time step for ions above and below $90 \mathrm{keV}$, respectively. The entire event is found to take place within less than 30 minutes. Despite the large distance from the Earth, the event appears to be a highly organized flux tube. The higher energy particles are observed throughout the event, while the lower energies with their smaller gyroradii are found only in the center.

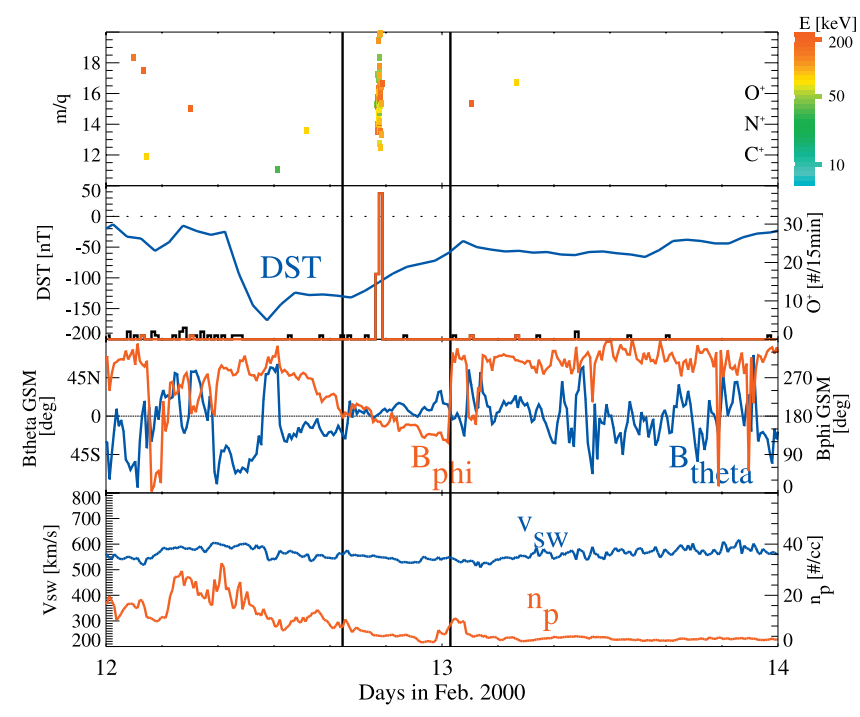

Figure 1. Observations on Feb 12 and 13, 2000 of suprathermal ions with $\mathrm{m} / \mathrm{q}>10$ by Wind/SMS/STICS (top two panels). The red and black histograms in the second panel show the number of LCSHI and instrumental background counts, formed by solar wind protons, respectively. The Dst index (second panel), the Wind/MFI interplanetary magnetic field azimuthal (red) and polar angle (blue) in GSM coordinates (third panel) and the Wind/ SWE solar wind speed (blue) and density (red) are shown in the bottom panel. The magnetic cloud passage of Wind is indicated by black vertical lines.

Above the panel the average flow angles in the ecliptic (upper row, $X_{\mathrm{GSE}}$ up, $\mathrm{Y}_{\mathrm{GSE}}$ to the right), and out-of-plane ( $\mathrm{Z}_{\mathrm{GSE}}$ up, $\mathrm{X}_{\mathrm{GSE}}$ to the left) are indicated with red and green lines for each time interval for the two specified energy ranges. Black arrows contain both energy ranges.

[10] On the right hand side another representation of the in-ecliptic flow angle is shown. Particles are found streaming away from the Earth, but not parallel to the interplanetary magnetic field line, which is indicated in blue. Particle streaming off field lines is interpreted as cross-field diffusion for the case of a gyrotropic distribution of particles. In this case, however, the condition of gyrotropy is not fulfilled, since the underlying flux tube is of limited extent and spatially structured. In fact, the flow angle with respect to the magnetic field direction and positive charge sign of particles indicates the gyromotion of the particles within the flux tube if the spacecraft moves across it north of its center axis. Another relevant piece of information is displayed in the small filled histogram at the bottom of the panel. Here the variances of the out-of-plane flow angles, derived from a minimum number of six particles per accumulation interval in the high energy bin, are given. These variances show a clear dependency on the distance from the center of the flux tube with minimum values at the boundary layers.

[11] For the numerical simulation shown in Figure 3, top, we used comparable values of pitch angle and magnetic field magnitude for the two specified energy ranges. The gyroradii of the ions shown here are, on average, $1.3 \mathrm{R}_{\mathrm{e}}$ (green) and $2.44 \mathrm{R}_{\mathrm{e}}$ (red). The resulting geometry of the flux tube, the counterclockwise motions of $\mathrm{O}^{+}$ions and the relative spacecraft motion are represented in a projection 


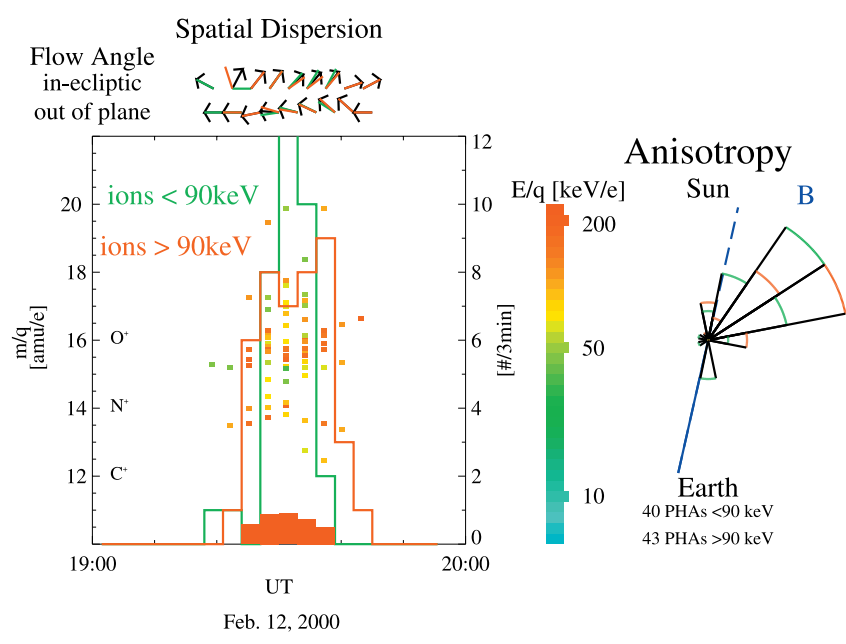

Figure 2. On the left is one hour of observations of Wind/ SMS/STICS data on Feb 12, 2000, starting at 1900 UT. Shown are the $\mathrm{m} / \mathrm{q}$ values and energy (colorscale) of individual ions over time, as well as the total number of $\mathrm{O}+$ and $\mathrm{N}+$ ions below $90 \mathrm{keV}$ (green line) and above $90 \mathrm{keV}$ (red line). The mean flow angles (azimuthal and polar) for all of the ions and separately for the two energy ranges are shown across the top of the panel. The relative variance (arbitrary units) of the polar angle is given by the red filled histogram along the bottom of the panel. On the right is the anisotropy of the particle flow in the spacecraft frame with respect to the average magnetic field direction (blue line), with the green and red arcs indicating the relative number of particles seen in that sector for ions below and above 90 $\mathrm{keV}$, respectively.

on the plane perpendicular to GSEx at the distance of the Wind spacecraft. The escaping magnetospheric ions are injected on the field line in the axis of the flux tube by assuming random gyrophases at the reconnection site. As Wind moves across the structure entering from approximately $10 \mathrm{~h}$ (the clock angle where Wind enters the flux tube, as seen on a cross sectional cut) it would initially observe the high energy fraction of particles moving approximately in the negative GSEy direction. Wind's position in the GSE frame is almost stationary, with a motion of less than $0.1 \mathrm{R}_{\mathrm{e}}$ for the time period the flux tube is observed. In this frame, the (dawnward) flux tube motion is three orders of magnitude larger than the velocity of the spacecraft. We assume that the flux tube motion is defined as the speed of a presumed path of reconnected field lines over time projected on a plane at 130 Re upstream. Note that the projected gyromotion of the particles is still about one order of magnitude larger than the (dispersive) motion of the (center of mass of the) flux tube. In the center of the structure particles show a larger variance with respect to their motions in the GSEy/GSEz plane. Wind leaves the flux tube at $3 \mathrm{~h}$ clock angle with particles moving in the positive GSEz direction. Thus our diagram in Figure 3 is consistent with the observations in Figure 2, indicating that the isolated burst of LCSHIs is from a single reconnected flux tube. The bottom of Figure 3 sketches, in 2D, the projected path of a $150 \mathrm{keV}$ singly charged oxygen ion along the large-scale interplanetary magnetic field geometry for the given relative locations of Wind and the Earth.
[12] With the observed motions of flux tube particles and the spatial distribution of high- and low-energy ions of the average gyroradii $2.44 \mathrm{R}_{\mathrm{e}}$ and $1.57 \mathrm{R}_{\mathrm{e}}$ within the flux tube it is possible to give an estimate for the scale size of the reconnection site. Assuming, for simplicity, an axissymmetric reconnection region in the center of the flux tube, its radius can be derived, using simple geometrical considerations, as $4.88(+1.0 /-1.5) R_{\mathrm{e}}$.

\section{Conclusions}

[13] On Feb. 12, 2000 the Wind spacecraft observed a flux tube containing magnetospheric origin ions. This flux tube was embedded in the magnetic cloud of an ICME. In situ magnetic field observations show that a direct magnetic connection was likely established from $130 \mathrm{R}_{\mathrm{e}}$ upstream all the way back to the Earth. The particles are spatially structured according to their kinetic energy with ions of small gyroradius in the center of the structure. Furthermore, the particles are highly non-gyrotropic and observed to
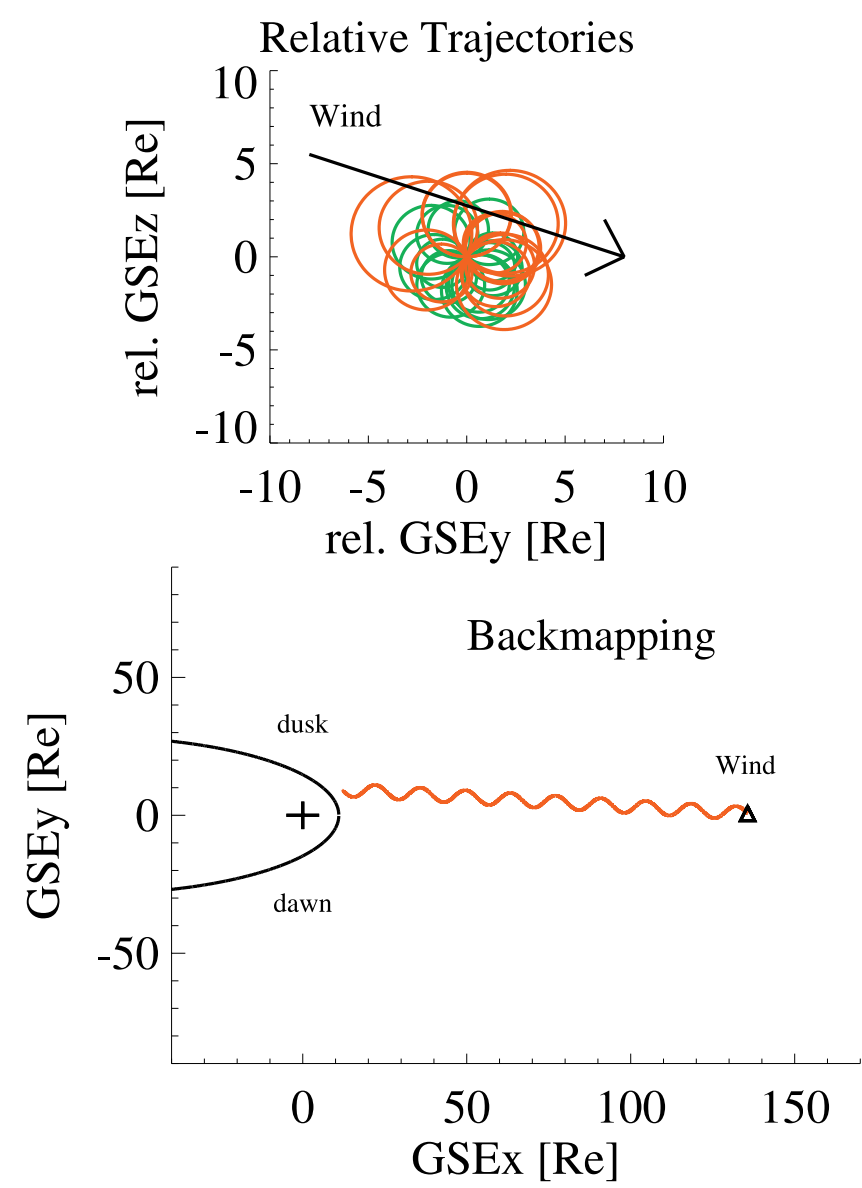

Figure 3. On top, the sample geometry of a flux tube with highly ordered spatial structuring is shown in a numerical simulation. Relative trajectories of the spacecraft and $\mathrm{O}^{+}$ ions with respect to the flux tube are projected on the plane perpendicular to GSEx. Note that ions have a counterclockwise motion along these circles. At bottom, the large scale geometry of the flux tube is shown for the relative geometries of Wind and the Earth. The projected gyromotion of $\sim 150 \mathrm{keV} \mathrm{O}^{+}$ions along the interplanetary magnetic field line is shown in scale. 
stream towards the Sun. We conclude that the LCSHI ions observed stream nearly scatter-free in the smooth field of the magnetic cloud. The differential intensity spectrum of $\mathrm{O}^{+}$and $\mathrm{N}^{+}$during the interval is almost flat in the energy range $3-13 \mathrm{keV} / \mathrm{amu}(50-200 \mathrm{keV} / \mathrm{e})$. The $\mathrm{O}+/ \mathrm{N}+$ ratio is $\sim 3.2$ in this energy range. Therefore, spectral and composition characteristics of the observed flux tube are roughly in agreement with observations of the storm time ring current [see Table 3 and Figure 13 in Gloeckler and Hamilton, 1987]. The fact that the observations coincide in time with the main recovery phase of the Earth's ring current, we conclude that the flux tube consists of ring current material flowing out at the dayside boundary layer. Given the slightly southward IMF it appears very likely that reconnection of the IMF with the magnetosphere favors this process.

[14] This case study complements the study of Posner et al. [2002] by describing magnetospheric outflow under circumstances of quiet magnetic field conditions. Typically, these flow out losses are found to be present in the fast solar wind with its highly variable magnetic field conditions. Therefore, the observations shown here offer the unique opportunity to study the physics of magnetospheric ion outflow into the upstream solar wind. Our preliminary estimate of the scale size of the flux tube, derived from the observed parameters, gives a radius of $4.88(+1.0 /-1.5)$ $\mathrm{R}_{\mathrm{e}}$. This can be regarded as an upper limit for the radius of the reconnection region at the dayside magnetopause. A 3D MHD simulation is currently preparation in order to trace back the observed flux tube into the magnetosphere.

[15] Acknowledgments. The authors are grateful for the assistance provided by J. Raines.

\section{References}

Christon, S. P., M. I. Desai, T. E. Eastman, G. Gloeckler, S. Kokubun, A. T. Y. Lui, R. W. McEntire, E. C. Roelof, and D. J. Williams, Low-Charge-
State Heavy Ions upstream of Earth's bow shock and sunward flux of ionospheric $\mathrm{O}^{+}, \mathrm{N}^{+}$, and $\mathrm{O}^{2+}$ ions: Geotail observations, Geophys. Res. Lett., 27, 2433-2436, 2000.

Desai, M. I., G. M. Mason, J. R. Dwyer, J. E. Mazur, T. T. von Rosenvinge, and R. P. Lepping, Characteristics of energetic ( $>30 \mathrm{keV} /$ nucleon) ions observed by the Wind/STEP instrument upstream of the Earth's bow shock, J. Geophys. Res., 105, 61-78, 2000.

Gloeckler, G., et al., The solar wind and suprathermal ion composition investigation on the Wind spacecraft, Space Sci. Rev., 71, 99, 1995.

Gloeckler, G., and D. C. Hamilton, AMPTE ion composition results, Physica Scripta, T18, 73, 1987.

Lepping, R. P., J. A. Jones, and L. F. Burlaga, Magnetic field structure of interplanetary magnetic clouds at 1 AU, J. Geophys. Res., 95, 11957, 1990.

Lepping, R. P., et al., The Wind Magnetic Field Investigation, Space Sci. Rev., 71, 207, 1995

Lepri, S. T., T. H. Zurbuchen, L. A. Fisk, I. G. Richardson, H. V. Cane, and G. Gloeckler, Iron charge distribution as an identifier of interplanetary coronal mass ejections, J. Geophys. Res., 106, 29,231, 2001.

Liemohn, M. W., J. U. Kozyra, V. K. Jordanova, G. V. Khazanov, M. F. Thomsen, and T. E. Cayton, Analysis of early phase ring current recovery mechanisms during geomagnetic storms, Geophys. Res. Lett., 25, 2845, 1999.

Möbius, E., D. Hovestadt, B. Klecker, M. Scholer, F. M. Ipavich, C. W. Carlson, and R. P. Lin, A burst of $\mathrm{O}^{+}$ions during an upstream particle event, Geophys. Res. Lett., 13, 1372-1375, 1986.

Ogilvie, K. W., et al., SWE, a comprehensive plasma instrument for the Wind spacecraft, Space Sci Rev., 71, 55, 1995.

Posner, A., N. A. Schwadron, T. H. Zurbuchen, J. U. Kozyra, M. W. Liemohn, and G. Gloeckler, Association of Low-Charge-State Heavy Ions up to $200 \mathrm{Re}$ upstream of the Earth's bow shock with geomagnetic disturbances, Geophys. Res. Lett., 29, doi:10.1029/2001GL013499, 2002.

Tsurutani, B. T., and W. D. Gonzalez, The cause of high intensity longduration continuous AE activity (HILDCAAs): Interplanetary Alfvn wave trains, Planet. Space Sci., 35, 405, 1987.

Williams, D. J., et al., GEOTAIL energetic particles and ion composition experiment, J. Geomag. Geoelectr., 46, 39, 1994.

Young, D. T., H. Balsiger, and J. Geiss, Correlations of magnetospheric ion composition with geomagnetic and solar activity, J. Geophy. Res., 87, 9077, 1982.

A. Posner, IEAP, University of Kiel, Leibnizstr. 11, 24118, Kiel, Germany. (posner@physik.uni-kiel.de)

M. W. Liemohn and T. H. Zurbuchen, Department of Atmospheric, Oceanic and Space Sciences, University of Michigan, 2455 Hayward, Ann Arbor, MI 48109, USA. (liemohn@umich.edu; thomasz@umich.edu) 